\title{
DETECÇÃO DE DANO EM PLACAS DE ALUMÍNIO UTILIZANDO IMPEDÂNCIA ELETROMECÂNICA
}

\author{
ANTONIO E. TURRA ${ }^{1}$, FABRICIO GUIMARÃES BAPTISTA ${ }^{2}$, VICENTE LOPES JR. ${ }^{1}$, JOZUÉ VIEIRA FI- \\ $\mathrm{LHO}^{3}$
}

1. Faculdade de Engenharia de Ilha Solteira, UNESP - Univ Estadual Paulista, Departamento de Engenharia Mecânica

A. Brasil, 56, 15.385-000 Ilha Solteira, São Paulo, Brasil

E-mails: turra@dem.feis.unesp.br, vicente@dem.feis.unesp.br

\author{
2. Faculdade de Engenharia de Bauru, UNESP - Univ Estadual Paulista, Departamento de Engenharia \\ Elétrica \\ Av. Eng. Luiz Edmundo C. Coube 14-01, Bairro: Vargem Limpa, 17033-360 Bauru, São Paulo, Brasil \\ E-mails: fabriciogbefeb.unesp.br
}

\author{
3. Faculdade de Engenharia de Ilha Solteira, UNESP - Univ Estadual Paulista, Departamento de En- \\ genharia Elétrica \\ A. Brasil, 56, 15.385-000 Ilha Solteira, São Paulo, Brasil \\ E-mails: jozueddee.feis.unesp.br
}

\begin{abstract}
This work proposes techniques for structural health monitoring based on indices to detect structural damages. Structural problems can cause accidents and it is extensively reported by the media. Some of these accidents result in fatalities, so, it is evident the importance of developing reliable methods for monitoring the structural integrity to prevent disasters resulting in loss of lives, environmental damage or economic losses. Therefore, it is of great technological interest and there are many research groups working with this topic. The present work deals with the implementation of a methodology for Structural Health Monitoring, SHM, based on H_2 norm_, Root-Means-Square Deviation (RMSD), Fault Index Metric (FIM), and Correlation Coefficient Deviation Metric(CCDM). The methodology is based on the variation of these indices in the healthy condition (baseline) and the monitored structural condition. It can give information about structural damages.
\end{abstract}

Keywords_ Intelligent Materials, Structural Health, Electromechanical Impedance, SHM.

Resumo- Este trabalho consiste em investigar técnicas de monitoramento de integridade estrutural baseadas em Índices de Falha para detectar danos estruturais. Inúmeros problemas estruturais intensamente noticiados pela mídia, alguns resultando em vítimas fatais, demonstram a importância de se desenvolver metodologias confiáveis de monitoramento da integridade estrutural a fim de evitar catástrofes que resultem em perdas de vidas humanas, danos ao meio ambiente ou prejuízos financeiros. Trata-se, portanto, de um tema atual e de grande interesse tecnológico, em virtude das questões econômicas e de segurança. Em particular, o trabalho consiste em estudar, implementar e aplicar uma metodologia de monitoramento da condição estrutural (do inglês Structural Health Monitoring, SHM) baseado em medidas da norma $\mathrm{H}_{2}$, RMSD (Root-Means-Square Deviation), IFM (Índice de Falha Métrica) e CCDM (Correlation Coefficient Deviation). A metodologia é baseada no cálculo de uma das normas para a estrutura com e sem falha e na avaliação da diferença entre elas. Assim, o dano estrutural é detectado.

Palavras-chave— Dinâmica Materiais inteligentes, integridade estrutural, impedância eletromecânica, SHM.

\section{Introdução.}

Muitas estruturas de engenharia mecânica, civil ou aeronáutica continuam sendo utilizadas apesar do grande tempo de vida e da possibilidade de apresentarem danos acumulados; ou mesmo contendo erros de projeto que poderão originar defeitos futuros, como trincas. Portanto, a habilidade de monitorar a condição destes sistemas, ou seja, sua integridade estrutural, tem se tornado uma área de pesquisa muito importante do ponto de vista econômico e de segurança nos últimos anos. Esta área é usualmente denominada de Structural Health Monitoring, SHM.
Exemplos de danos estruturais ocorridas no Brasil e no exterior demonstram a importância de se desenvolver metodologias confiáveis de SHM a fim de evitar catástrofes que possam resultar em perda de vidas humanas, prejuízos econômicos e danos ao meio ambiente. Assim, um maior avanço na tecnologia de sensores e nos procedimento de decisão com confiabilidade do diagnóstico do dano, se possível em um estágio inicial, são pontos fundamentais para tornar o SHM economicamente viável para estes tipos de aplicações e assim se evitar tais catástrofes. 


\section{O Monitoramento da Condição Estrutu- ral (Structural Health Monitoring).}

A maioria das aplicações reais de SHM se baseia, atualmente, em métodos visuais e experimentais localizados, tais como os métodos acústicos ou ultrasônicos, métodos de campo magnético, métodos de radiografia e termografia, (Doherty, 1987). Todos estes métodos exigem que a proximidade do dano seja conhecida a priori e que a parte da estrutura a ser inspecionada seja prontamente acessível. Com estas limitações, estes métodos experimentais podem detectar danos apenas superficiais ou muito próximos à superfície. Assim, a necessidade de um processo de SHM global para diagnóstico de falhas que possa ser aplicado em estruturas complexas tem motivado pesquisas que examinam mudanças nas propriedades de vibração da estrutura. Este tema tem recebido considerável atenção na literatura técnica e as últimas pesquisas têm sido direcionadas para o desenvolvimento de fundamentos físicos e matemáticos para esta tecnologia.

A ideia básica do problema SHM, baseado em sinais de vibração, é que a presença de danos altera as propriedades físicas do sistema - por exemplo, redução de rigidez causada pela presença de trincas e/ou provoca variações das propriedades geométricas que podem vir a mudar as condições de contorno e a conectividade do sistema. Todos estes fatores podem comprometer o desempenho futuro da estrutura e resultar em uma mudança na resposta dinâmica do sistema. Portanto, na definição de falha está posto que o problema de diagnóstico é uma comparação entre dois estados de um sistema: um estado sem falha e outro estado com falha.

O SHM é definido como um processo de implementação de uma estratégia de decisão para detecção de danos em estruturas de engenharia civil, mecânica ou aeroespacial. Este processo envolve: a observação das medidas de resposta dinâmicas de uma estrutura, que podem ser obtidas através de uma grande diversidade de sensores; a extração de indicadores sensíveis ao dano a partir destas medidas e a análise destes indicadores para determinar a condição real do sistema em questão.

Um dos desafios fundamentais de um processo de SHM vem do fato de que um dano estrutural é tipicamente um fenômeno local e pode não influenciar significativamente a resposta global da estrutura, principalmente nas baixas frequências, que são normalmente excitadas e medidas durante os testes de vibração, Farrar et al. (2000). Entretanto, a popularização dos materiais inteligentes nos últimos anos, tem possibilitado o monitoramento de impedância elétrica para SHM. Uma vez que a impedância elétrica é adquirida em altas frequências, ela provém informações sobre modos locais, ou seja, permite a detecção de danos incipientes que ainda não influenciaram significativamente a resposta global, (Lopes Jr. et al., 2000, Furtado e Lopes Jr., 2004). Outra restrição dos algoritmos de SHM é que muitos deles devem ser implementados em um modo de aprendizagem não-supervisionado, o que implica que os dados do sistema com dano não são disponíveis. Isso é verdade para a grande maioria das aplicações em estruturas reais, conforme descrito em Fugate et al. (2000). Assim, as pesquisas mais recentes de SHM, baseadas em medidas de vibração, passaram a ser descritas dentro do paradigma de reconhecimento estatístico de padrões. A falta de análise estatística apresenta um potencial problema para o desenvolvimento da tecnologia de detecção de danos, baseada em medidas de vibração, para aplicações reais (Farrar e Sohn, 2000 e Farrar et al., 2003).

\section{Métodos Baseados na Impedância Elétri-} ca.

Elementos piezelétricos têm sido usados com sucesso para monitoramento de estruturas e em projetos de controle ativo para diferentes sistemas, tal como vigas, placas, treliças, etc. (Dosh et al., 1992).

Embora existam diversos trabalhos apresentando a utilização de parâmetros modais na detecção e localização de danos estruturais, o emprego desta metodologia não é trivial e apresenta alguns obstáculos. Um destes obstáculos é o fato de que um dano é um fenômeno local, enquanto, as informações modais refletem as informações globais do sistema. Neste sentido, utiliza-se o método da impedância elétrica para detectar e localizar os danos em uma estrutura. $\mathrm{O}$ método emprega atuadores/sensores piezelétricos (PZT) para excitar/medir a impedância elétrica em altas faixas de frequência (tipicamente acima de 10 $\mathrm{kHz}$ ). As altas frequências limitam a área de influência de cada PZT, permitindo trabalhar com os modos locais, avaliando, assim, apenas as informações correspondentes à área de atuação de cada PZT. O princípio básico consiste em monitorar a variação da impedância mecânica, causada devido à presença de uma mudança estrutural (dano), através da impedância elétrica.

A técnica baseada na impedância utiliza o PZT como atuador e sensor. Um PZT colado na estrutura e alimentado por um campo elétrico alternado com valor fixo excita e induz vibrações na estrutura (efeito inverso). A vibração resultante gera um fluxo de corrente no PZT (efeito direto), que é função do grau de interação mecânica entre o PZT e a estrutura, dentro da faixa de frequência considerada.

Um modelo simples, como mostrado na figura 1, pode descrever a interação entre um PZT e a estrutura a ele vinculada. O PZT é considerado como uma barra fina exposta a uma vibração axial em resposta a aplicação de tensão elétrica alternada. Uma extremidade é fixa e a outra é conectada na estrutura, representada por um sistema de um grau de liberdade.

Na Figura 1, $M$ representa a massa, $K$ é a constante elástica da mola e $\mathrm{C}$ é o coeficiente de amorte- 
cimento. O transdutor é excitado por uma fonte de voltagem senoidal com amplitude $v$ e uma frequencia angular $\omega$ que produz a corrente $I$ com amplitude $i$ e fase $\varphi$. Considerando que as propriedades do transdutor é invariante no tempo, qualquer mudança na estrutura irá afetar o valor da impedância do sistema.

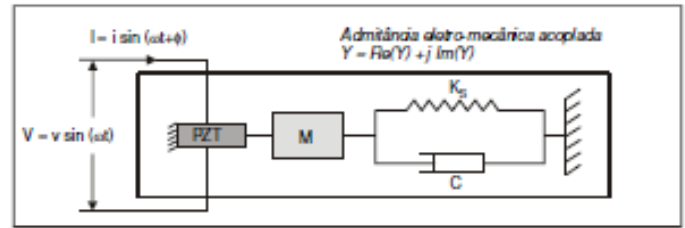

Figura 1. Modelo usado para representar o acoplamento de um PZT com a estrutura base (Park,2003).

A solução do sistema mostrado na figura $1 \mathrm{em}$ ternos da impedância elétrica do transdutor é dada de acordo com (Liang, 1994) pela seguinte equação:

$$
Z_{E}(\omega)=\frac{U}{I}=\frac{i}{j \omega a}\left(\varepsilon_{33}^{T}-\frac{Z(\omega)}{Z(\omega)+Z_{a}(\omega)} d_{3 x}^{2} \hat{Y}_{x x}^{E}\right)^{-1}
$$

Na Equação (1) $U$ representa a voltagem de entrada e $I$ a corrente elétrica de saída do transdutor piezelétrico. Além disso, $a$ é uma constante geométrica e, $d_{3 x}^{2}, \hat{Y}_{x x}^{E}$ e $\varepsilon_{33}^{T}$ são, constantes do acoplamento piezelétrico, modulo de Young, e a constante dielétrica complexa do transdutor piezelétrico em uma voltagem constante, respectivamente.

De acordo com a equação (1), qualquer mudança na impedância mecânica da estrutura provocada por dano implica em uma variação correspondente na impedância elétrica do transdutor. Portanto, a técnica da Impedância Eletromecânica ( EMI ) possibilita a avaliação da integridade estrutural de uma maneira simples pela medida da impedância elétrica do transdutor PZT.

\section{4 Índices de Falha}

Os quatro índices obtidos através da Impedância Eletromecânica investigados neste trabalho estão brevemente descritos a seguir. A detecção de falhas é baseada em uma comparação entre a Impedância Eletromecânica da estrutura na condição saudável e da estrutura em condições normais de operação (condição desconhecida).

\subsection{Root-Means-Square Deviation (RMSD)}

O índice RMSD é apresentado da seguinte forma (Lopes Jr. et al., 2000):

$$
R M S D=\sum_{i=1}^{n} \sqrt{\frac{\left[\left(Y_{i, S F}\right)-\left(Y_{i, C D}\right)\right]^{2}}{\left[\left(Y_{i, S F}\right)\right]^{2}}}
$$

em que $Y_{i, S F}$ é a magnitude da impedância medida no sinal de referência (baseline), ou seja, da estrutura saudável, obtida através do PZT sensor e $Y_{i, C D}$ é a magnitude da impedância do sinal do mesmo PZT medida em condições estruturais desconhecidas no intervalo de frequência $i$. n é o número de pontos utilizados na aquisição do sinal. (Silva et al., 2008).

\section{2 $\mathrm{Norma} \mathrm{H}_{2}$}

Índices de Falha são como medidas de tamanho e podem ser utilizadas para diversas aplicações. A norma $\mathrm{H}_{2}$ de um sistema é usada para caracterizar o sistema em si, juntamente com os seus modos e seus sensores. Sendo $G(\omega)$ uma função de transferência de um sistema, a norma $\mathrm{H}_{2}$ do sistema é definida como segue (Gawronski, 1998):

$$
\|G\|_{2}^{2}=\frac{1}{2 \pi} \int_{-\infty}^{+\infty} \operatorname{tr}\left(G^{*}(\omega) G(\omega)\right) d \omega
$$

em que tr é o traço de $G^{*}(\omega) \mathrm{G}(\omega)$.

Geralmente, a norma $\mathrm{H}_{2}$ é calculada utilizando coordenadas modais, mas desta forma é necessário obter um modelo para a equação proposta. $\mathrm{O}$ valor numérico da norma $\mathrm{H}_{2}$ para um sistema SISO (única entrada e única saída) corresponde à área abaixo da Impedância Eletromecânica do sistema (Gawronski, 1998). Em situações práticas, a obtenção do modelo matemático pode ser difícil, por isso, para superar esta situação, pode-se considerar a área abaixo da curva da impedância. Sendo assim, a área abaixo da curva da impedância foi computada utilizando o método trapezoidal, implementado no software MA$\mathrm{TLAB}^{\circledR}$ através do comando "trapz".

A norma $\mathrm{H}_{2}$ pode ser utilizada para detecção de falhas segundo o procedimento: considera-se a norma computada utilizando o jésimo PZT atuador / sensor, e denota $\left\|G_{S F j}\right\|_{2}^{2}$ para a estrutura saudável e $\left\|G_{C D j}\right\|_{2}^{2}$ para a estrutura em uma condição estrutural desconhecida. O índice de falha referente ao jésimo sensor $\left(\mathrm{IH}_{2}\right)$ é definido como a diferença ponderada entre a norma computada em uma estrutura saudável e a norma computada em uma estrutura em condições estruturais desconhecidas (Gawronski e Sawicki, 2000), como segue:

$$
I H_{2}=\frac{\left\|\left|G_{S F j}\left\|_{2}^{2}-\right\| G_{C D j} \|_{2}^{2}\right|\right.}{\left\|G_{S F j}\right\|_{2}^{2}}
$$


em que $j=1, \ldots, \mathrm{r}$; e $\mathrm{r}$ é o número do PZT atuador/sensor.

\section{3 Índice de Falha Métrica (IFM)}

A impedância entre duas condições estruturais diferentes pode também ser comparada através do Índice de Falha Métrica (IFM), que fornece uma estimativa da variação estrutural, como mostrado na seguinte equação.

$$
I F M=\sum_{i=1}^{n}\left[\left(Y_{i, S F}\right)-\left(Y_{i, C D}\right)\right]^{2}
$$

em que $I F M$ é o Índice de Falha Métrica, $Y_{i, S F}$ é a magnitude da impedância medida na estrutura saudável, $Y_{i, C D}$ é a magnitude da impedância medida em condições normais de funcionamento da estrutura (condição desconhecida) no intervalo de frequência $i$; n é o número de pontos utilizados na aquisição do sinal.

\subsection{Correlation Coefficient Deviation (CCDM):}

O índice CCDM é dado por:

$$
\begin{gathered}
\mathrm{I}_{\mathrm{CCDM}}=1-\rho=1-\frac{\operatorname{cov}\left(Y_{S F}, Y_{C D}\right)}{S_{Y_{S F}} S_{Y_{C D}}} \\
\mathrm{I}_{\mathrm{CCDM}=}=1-\frac{1}{n-1} \frac{\sum_{i=1}^{n}\left(Y_{i, S F}-\bar{Y}_{S F}\right)\left(Y_{i, C D}-\bar{Y}_{C D}\right)}{S_{Y_{S F}} S_{Y_{C D}}}
\end{gathered}
$$

em que $\rho$ é o coeficiente de correlação, cov é a covariância cruzada e $S$ é relativo ao desvio padrão. $Y_{i, S F}$ é a impedância medida na estrutura saudável, $Y_{i, C D}$ é a impedância medida na estrutura em uma condição desconhecida, no intervalo de frequência $i$. $\bar{Y}$ é o valor médio da impedância para cada caso. $\rho$ indica o quão bem o baseline (sinal de referência) e as medições em condições desconhecidas estão linearmente relacionadas. Assim, valores elevados expressam que os dados não são correlacionados ou, então, que existe uma variação, provavelmente ocorrida devido a presença da falha na estrutura.

\section{APLICAÇÕES DA METODOLOGIA}

Para as medições de impedância utilizou-se um sistema de baixo custo desenvolvido em LabVIEW (Baptista e Vieira Filho, 2009). A figura 2 abaixo mostra o diagrama de blocos do sistema geral.

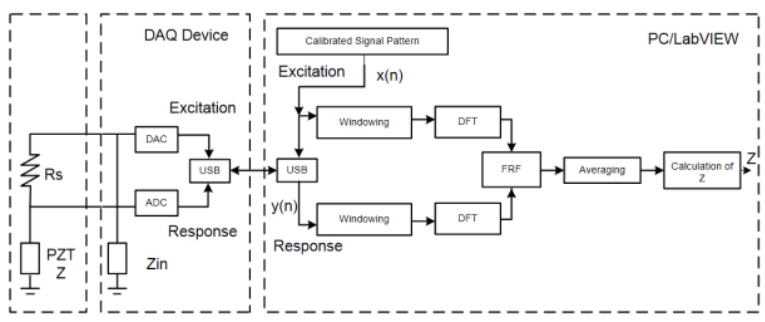

Figura 2. Sistema completo de medida de impedância com PZT.

O sistema utiliza um sistema de aquisição (DAQ) da National Instruments (NI). Através do software mencionado, foi gerado um sinal chirp para a excitação. Para garantir uma boa medição o sinal de excitação gerado e a aquisição da resposta ao sinal foram sincronizados antes das medições serem realizadas.

Os testes experimentais foram executados em placas retangulares biapoiadas em que foram introduzidas falhas com largura de $1 \mathrm{~mm}$ (estrutura A) e $2 \mathrm{~mm}$ (estruturas B e C) através de eletroerosão para garantir uma melhor exatidão das dimensões das falhas, conforme mostrados nas figuras 3, 4 e 5 .

Foram utilizados 2 sensores de PZT para excitar a estrutura e medir a resposta da impedância eletromecânica. As disposições destes sensores foram de tal forma para que se pudesse avaliar o efeito da distancia e a direção do sensor em relação à falha.

Foram utilizadas duas faixas de excitação: de 0 a $62,5 \mathrm{kHz}$ e 0 a $125 \mathrm{kHz}$ para avaliação da melhor faixa para este tipo de estrutura.

Neste artigo é apresentado testes experimentais onde avaliamos a estrutura sem dano e estrutura com falha e utilizaremos 4 índices para avaliação do dano - norma $\mathrm{H}_{2}$, RMSD, IFM e CCDM.

Estes índices são utilizados normalmente, segundo a literatura, para o uso da FRF como sinal. Avaliamos o uso da Magnitude da Impedância eletromecânica para as estruturas (placas) de alumínio (430x150x2 mm) das figuras abaixo. Foram introduzido falhas à $165 \mathrm{~mm}$ da borda.

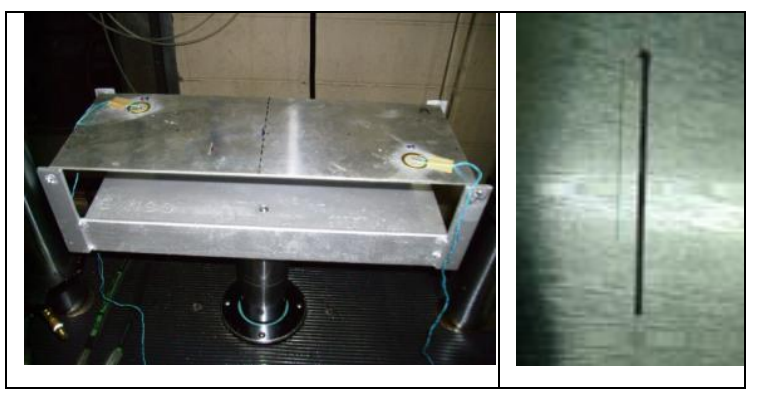

Figura 3 - Estrutura A, Falha 20x1 mm. 


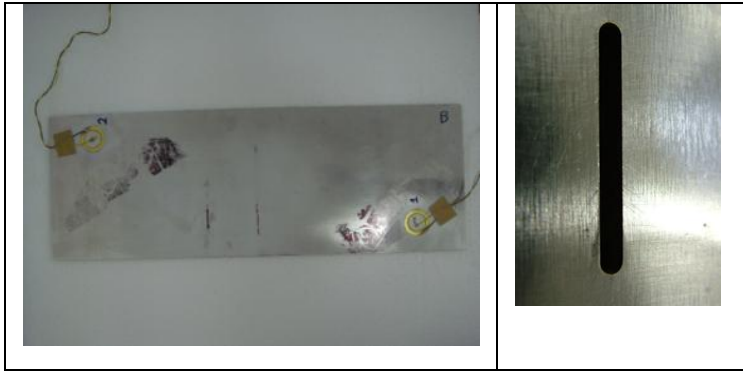

Figura 4 - Estrutura B, Falha 20x2 mm (terminação arredondada).

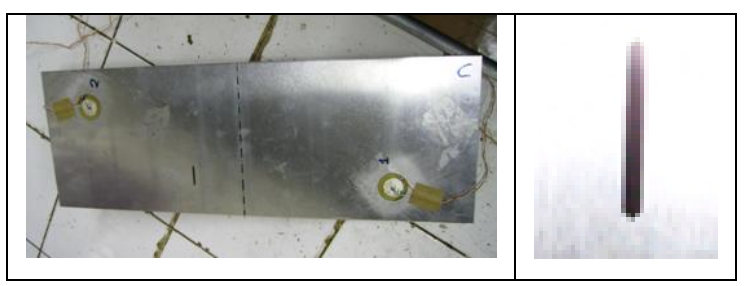

Figura 5 - Estrutura C, Falha 20x2 mm (terminação canto vivo).

Para as figuras abaixo o numero 1 refere-se ao sensor1 da estrutura A; 2 ao sensor2 de A; 4 e 5 ao sensor1 e sensor2 da Estrutura B; 7 e 8 ao sensor1 e sensor2 da Estrutura C, respectivamente e a faixa de frequências escolhidas para a resposta foi de 40 a 60 $\mathrm{kHz}$, utilizando a magnitude da impedância.
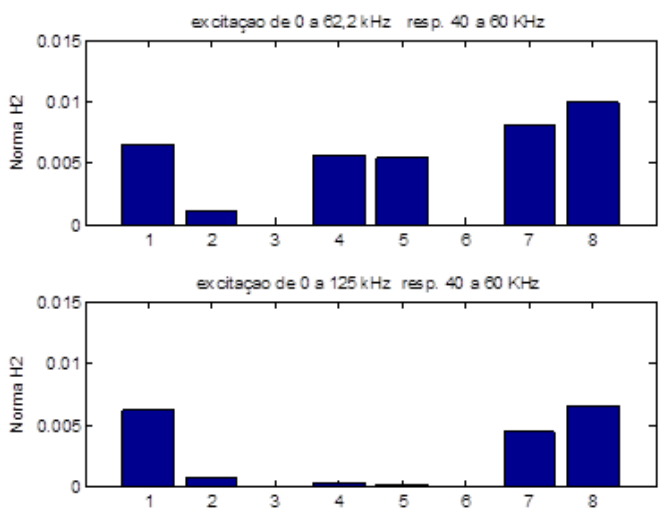

Figura 6 - Norma H2, resposta de 40 a $60 \mathrm{kHz}$.
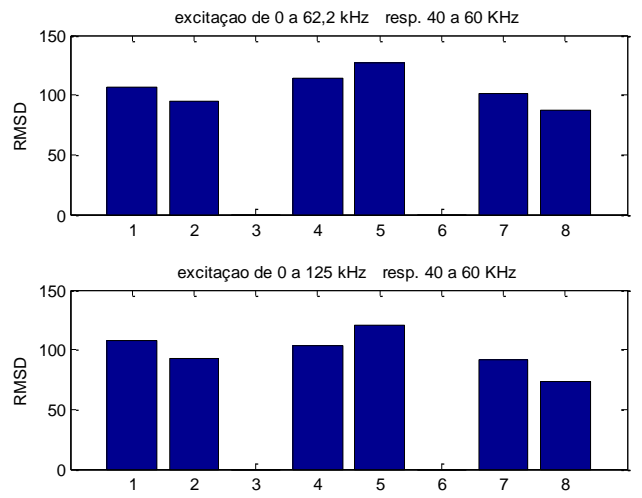

Figura 7 - Índice RMSD, resposta de 40 a 60 kHz.
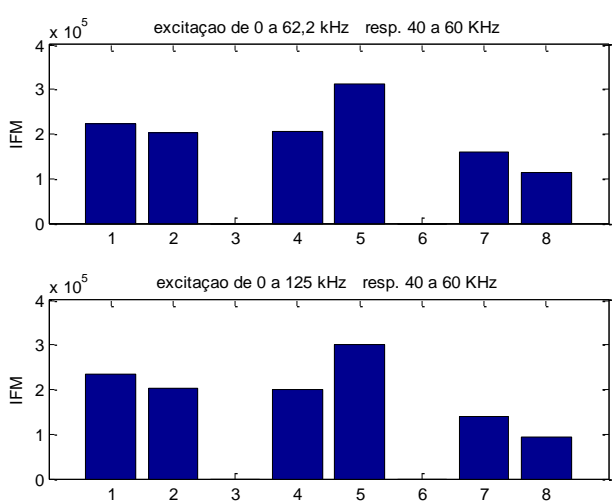

Figura 8 - IFM, resposta de 40 a $60 \mathrm{kHz}$.
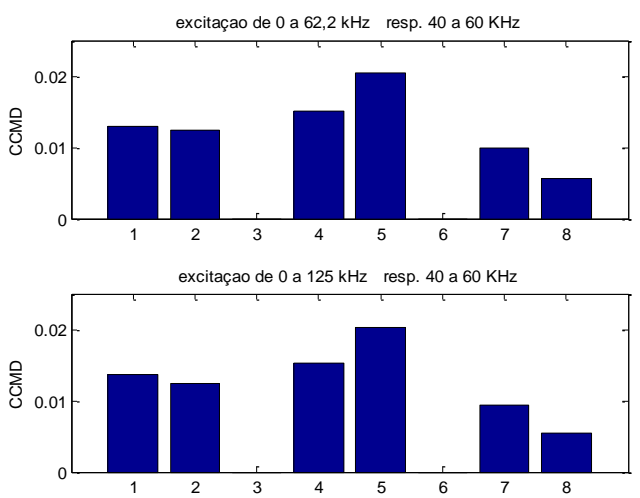

Figura 9 - Índice CCDM, resposta de 40 a $60 \mathrm{kHz}$.

Avaliamos também o comportamento dos índices de falhas para a faixa de excitação de 0 a 62,5 $\mathrm{kHz}$ e resposta para a faixa de 20 a $40 \mathrm{kHz}$, utilizando a magnitude da impedância como sinal da estrutura, cujos resultados são apresentados nas figuras abaixo.

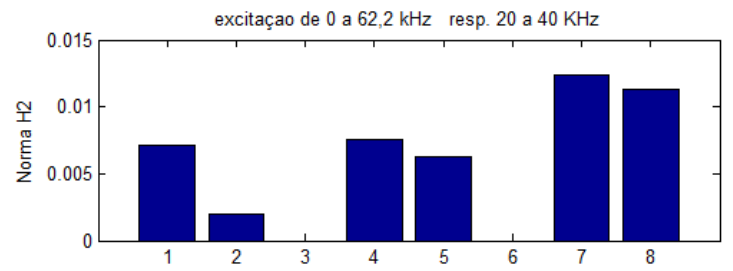

Figura 10 - Norma H2, resposta de 20 a $40 \mathrm{kHz}$.

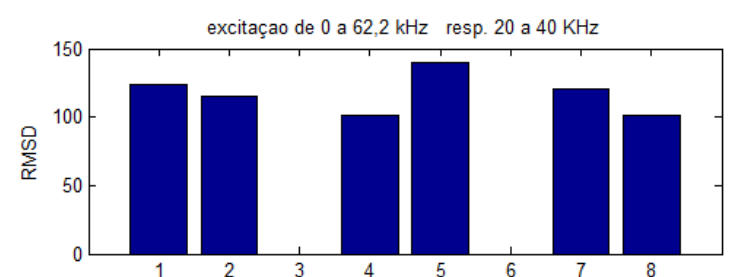

Figura 11 - Índice RMSD, resposta de 20 a 40 kHz. 


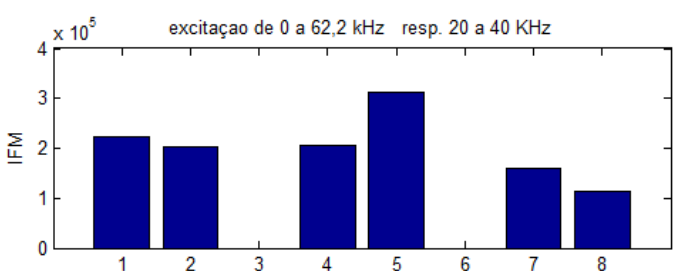

Figura 12 - IFM, resposta de 20 a $40 \mathrm{kHz}$.

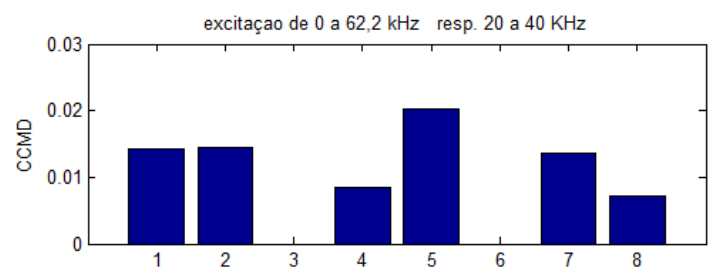

Figura 13 - Índice CCDM, resposta de 20 a 40 kHz.

\section{Conclusão}

$\mathrm{Na}$ avaliação do comportamento deste tipo de estrutura verificamos que a faixa de excitação, limitada pela placa de aquisição, teve um melhor comportamento para excitação entre 0 e $62,5 \mathrm{kHz}$ e que a utilização do valor da Magnitude da Impedância eletromecânica é bastante adequado.

A utilização dos índices de falhas detectaram as falhas introduzidas, com melhor resultado para a faixa de $20 \mathrm{kHz}$ a $60 \mathrm{kHz}$.

\section{Agradecimentos}

Os autores agradecem ao CNPq e à FAPEMIG pelo financiamento parcial deste trabalho, através do INCT-EIE.

\section{Referências Bibliográficas}

Baptista, F. G. and J. Vieira Filho, 2009. "New impedance measurement system for PZT based structural health monitoring," IEEE Transactions on Instrumentation and Measurement, New York, v. 58, n. 10, p. 3602-3608. DOI:

10.1109/TIM.2009.2018693

Doherty, J. E. "Nondestructive Evaluation", Chapter 12 in Handbook on Experimental Mechanics, A. S. Kobayashi Edt., Society fo Experimental Mechanics, Inc. 1987.

Dosh, J. J. e Inman, D. J., “A Self-Sensing Piezoelectric Actuator for Collocated Control", Journal of Intelligent Material Systems and Structures, vol. 3, pg. 166-185, 1993. DOI: 10.1177/1045389X9200300109

Farrar, C. R. and Sohn, H. "Pattern recognition for structural health monitoring". In: Workshop on
Mitigation of Earthquake Disaster by Advanced Technologies, Las Vegas,NV, USA, 2000.

Farrar, C. R. et al. "Damage prognosis: current status and future needs". In: Los Alamos National Laboratory Report, LA-14051-MS, 2003.

Fugate, M. L., Sohn, H. and Farrar, C. R. "Unsupervised learning methods for vibration-based damage detection". In: 18th International Modal Analysis Conference - IMAC, San Antonio, Texas, USA, 2000.

Furtado, R. M. and Lopes Jr., V. "Damage characterization in flexible structure through piezoelectric sensor and actuator". In: 11th International Congress on Sound and Vibration, St. Petersburg, Russia, p. 3393-3400, 2004.

Gawronski, W. Dynamics and control of structures: a modal approach, 1. Ed. New York: Springer Verlag, 1998. DOI: 10.1007/978-0-387-21855-7

Gawronski, W K.; Sawicki, J.T. Structural damage detection using modal norms. 2000

Liang, C.; Sun, F.P.; Rogers, C.A. An impedance method for dynamic analysis of active material system. Journal of Vibration and Acoustics, USA, v. 116, n..., p. 121-128, 1994.

Lopes JR. V.; Pereira, J.A.; Inman, D.J. Structural FRF acquisition via electric impedance measurement applied to damage location. In: International Modal Analysis Conference -IMAC, 18, 2000, Texas. Proceedings... Texas, 2000a. p. 1549-1555.

G. Park, H. Sohn, C. R. Farray and D. J. Inman, Nov 2003. "Overview of piezoelectric impedancebased health monitoring and path forward," The Shock and Vibration Digest, v. 35, n. 1, p. 451463. DOI: $10.1177 / 05831024030356001$

Silva, S.; Dias Junior, M.; Lopes Junior, V. Structural health monitoring in smart structures through time series analysis. Structural Health Monitoring, USA, v. 7, n. 3, p. 231-244, 2008. DOI: $10.1177 / 1475921708090561$ 